\title{
EBSD Usage in Grain Boundary Engineering of CFC Alloys
}

\author{
Andre Luiz Pinto \\ Carlos Sergio da Costa Viana
}

IME - Pca. Gen. Tiburcio, 80, Rio de Janeiro, Brazil, ZIP 22290-270, pinto@ime.eb.br

The concept of grain boundary engineering (GBE) has been proposed as a new way of modifying materials properties[1] through the control the grain boundary crystallography. Improvement in the creep resistance and resistance to corrosion using this concept has been reported[2]. In order to actually perform GBE, it is necessary characterize the grain boundary. The coincidence site lattice theory offers a simple and approximate treatment through the evaluation of the density of coincidence sites when the lattice of one grain is superposed to the lattice of its neighbor. The reciprocal of the density of these coincidence sites, $\Sigma$, is used to characterize the boundaries. These boundaries have shown special properties compared to random boundaries.

Different approaches were used to perform GBE on CFC materials. The generation of twins through recrystallization after moderate plastic deformation followed by cycles of low deformation and annealing seems promising. The same kind of treatment has been applied to different materials normalizing the recrystallization and annealing temperatures through the melting point, $\mathrm{T}_{\mathrm{M}}$. The materials used so far are $\alpha$-brass (Cu30Zn), commercially pure lead $(99,9 \%)$ and Inconel 600 (Ni17Cr9Fe). Cold rolling was used as the deformation method on the two first materials and swaging was used on the last one. The amount of deformation used on the recrystallization step was around $40 \%$ and recrystallization temperatures and times were calibrated for obtaining a grain size around 6 ASTM in each material. The low deformation used was around $6 \%$ and the annealing temperature was around $0,8 \mathrm{~T}_{\mathrm{M}}$. The EBSD technique has been used to rapidly characterize the amount of CSL boundaries and microtexture. The definition of a grain boundary as a CSL was done according to the Brandon criterion[3].

GBE was successfully done on these materials; the maximum amounts of CSL boundaries achieved on $\alpha$-brass, lead and Inconel 600 were respectively: $63 \%, 57 \%$ and $60 \%$. Except for the lead, where repetition of the low deformation-annealing cycle actually decreased the amount of CSL boundaries, the iterative treatments have proved effective. Figure 1 shows the orientation maps of $\alpha$-brass at the recrystallized state and after one low deformation-annealing cycle. Figure 2 shows orientation maps from the same conditions of lead and Inconel 600. In the samples submitted to the low deformation-annealing treatment there is a considerable amount of twins that do not cross the entire width of the grain. These twins have risen after the low deformation step. Figure 3 shows the misorientation ahead of the incoherent twin boundary in an $\alpha$-brass sample recrystallized and submitted to low deformation. The distortion of the crystal lattice observed provides the driving force for the growing of these twins. These treatments have also significantly improved creep resistance, although the mechanisms through which this is achieved remain unexplained.

[1] T. Watanabe, Res. Mechanica 11 (1984) 47.

[2] G. Palumbo et al., JOM 50(N. 2) (1998) 40.

[3] D. G. Brandon, Acta Metallurgica 14 (1966)1479.

[4] The aid of Dr. L. H. de Almeida and Dr. H. R. Z. Sandim is gratefully acknowledged. 


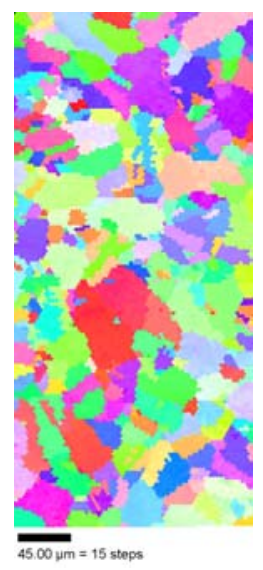

(a)

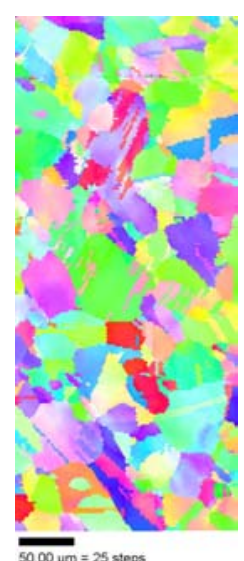

(b)

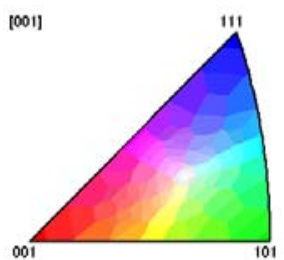

(c)

Figure 1 - Orientation maps of $\alpha$-brass at the (a) recrystallized state and (b) one cycle of low deformation-annealing treatment. (c) inverse pole figure reference map.

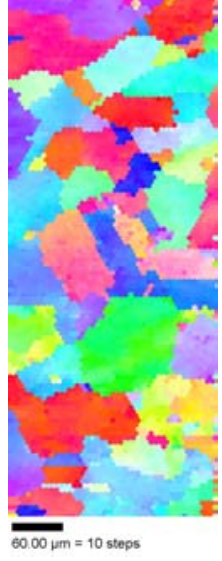

(a)

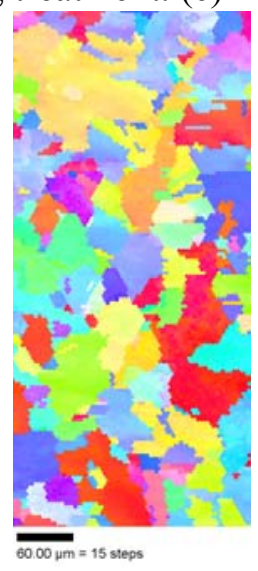

(b)

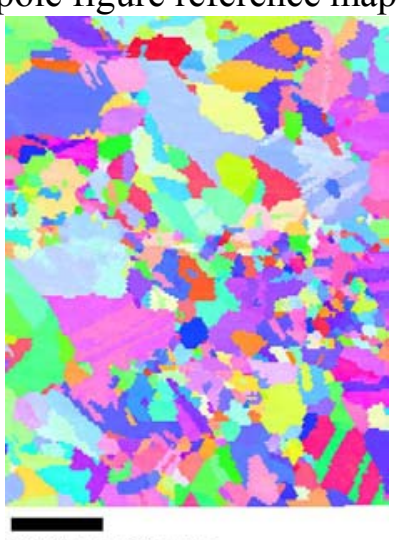

$30.00 \mu \mathrm{m}=30$ steps

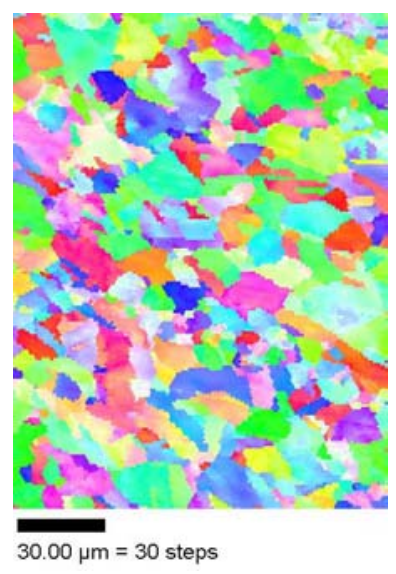

(d)

Figure 2 - Orientation maps of lead at the (a) recrystallized state and (b) one cycle of low deformation-annealing treatment. Orientation maps of Inconel 600 at the (c) recrystallized state and (d) one cycle of low deformation-annealing treatment.

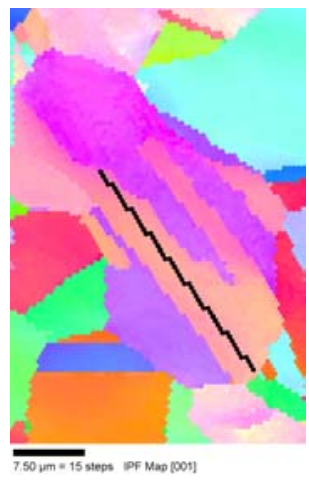

(a)

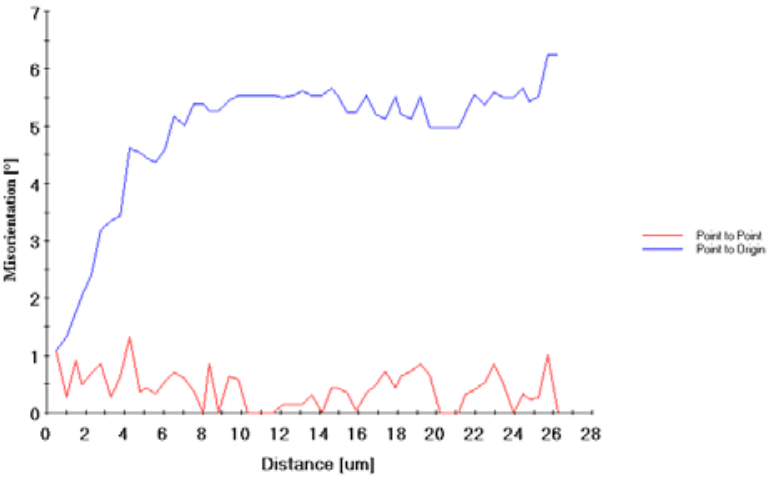

(b)

Figura 3 - (a) Orientation map of $\alpha$-brass submitted to recrystallization and low deformation before the annealing treatment; (b) Misorientation along the black line shown in (a). 